\title{
Waldschmidt constants for Stanley-Reisner ideals of a class of graphs
}

\author{
Tomasz Szemberg and Justyna Szpond
}

January 23, 2018

\begin{abstract}
In the present note we study Waldschmidt constants of Stanley-Reisner ideals of a hypergraph and a graph with vertices forming a bipyramid over a planar $n$-gon. The case of the hypergraph has been studied by Bocci and Franci. We reprove their main result. The case of the graph is new. Interestingly, both cases provide series of ideals with Waldschmidt constants descending to 1 . It would be interesting to known if there are bounded ascending sequences of Waldschmidt constants.
\end{abstract}

\section{Introduction}

The following problem has attracted considerable attention in commutative algebra and algebraic geometry in the past two decades.

Problem 1.1 (Containment problem). Let $I$ be a homogeneous ideal in the polynomial ring $\mathbb{K}\left[x_{0}, \ldots, x_{N}\right]$, where $\mathbb{K}$ is a field. Decide for which integers $m$ and $r$ there is the containment

$$
I^{(m)} \subset I^{r}
$$

between the symbolic and ordinary powers of the ideal $I$.

We recall that for $m \geqslant 0$ the $m$-th symbolic power of $I$ is defined as

$$
I^{(m)}=\bigcap_{P \in \operatorname{Ass}(I)}\left(I^{m} R_{P} \cap R\right),
$$

where $\operatorname{Ass}(I)$ is the set of associated primes of $I$. At the beginning of the Millennium, Ein, Lazarsfeld and Smith in characteristic zero [5] and Hochster and Huneke in positive characteristic [8] proved striking uniform answers to Problem 1.1 to the effect that the containment in (1) holds for all

$$
m \geqslant h r,
$$

where $h$ is the maximum of heights of all associated primes of $I$. In geometric terms it means that $h$ is the codimension of the smallest embedded component of the set Zeroes $(I)$. In particular, the containment in (1) holds for all $I$ with $m \geqslant N r$.

It is natural to wonder to what extend the particular bound in (3) is sharp. In order to study this question Bocci and Harbourne introduced in [3] a number of asymptotic invariants attached to $I$. In the present note we focus on one of them. Let $\alpha(I)$ denote the smallest degree of a non-zero element in $I$, this is the initial degree of $I$. Then, the Waldschmidt constant of $I$ is asymptotically defined as

$$
\widehat{\alpha}(I)=\lim _{m \rightarrow \infty} \frac{\alpha\left(I^{(m)}\right)}{m} .
$$

It is well known, see e.g. proof of [3, Lemma 2.3.1], that $\widehat{\alpha}(I)=\inf _{m \geqslant 1} \frac{\alpha\left(I^{(m)}\right)}{m}$. 
Interestingly, Waldschmidt constants were introduced long before the Problem 1.1 has been considered in the realms of complex analysis, see 10 and our note 9 for recent account. These invariants are very hard to compute in general. In fact, a number of important conjectures can be expressed in terms of Waldschmidt constants. By the way of an example we mention here only the following one.

Conjecture 1.2 (Nagata). Let $I$ be the ideal defining $s \geqslant 10$ very general points in $\mathbb{P}^{2}$. Then

$$
\widehat{\alpha}(I)=\sqrt{s} .
$$

Our research here has been motivated by [2, where Bocci and Franci initiated the study of Waldschmidt constants of monomial ideals determined by some combinatorial data. They have computed Waldschmidt constants of Stanley-Reisner ideals of bipyramids (see Section 2.2). We provide here an alternative, more elementary proof of their result and study Stanley-Reisner ideals of graphs determined by vertices of bipyramides. Our main result is Theorem 3.2 .

\section{Bipyramids revisited}

We begin by recalling briefly basic notions from combinatorial algebra relevant in this note, for more detailed account see the very nice surveys [6] and [7. The Stanley-Reisner ideals introduced here have traditionally provided a rich source of non-trivial examples.

\subsection{Stanley-Reisner theory}

Definition 2.1 (Simplicial complex). A simplicial complex $\Delta$ on a finite set $V$ is a collection of subsets $\sigma \subset V$ such that the containment $\sigma \in \Delta$ implies $\tau \in \Delta$ for all subsets $\tau \subset \sigma$.

For the set $V=\{0,1, \ldots, N\}$, we can naturally identify any subset $\sigma \subset V$ with a squarefree monomial

$$
x_{\sigma}=\prod_{i \in \sigma} x_{i} \in \mathbb{K}\left[x_{0}, \ldots, x_{N}\right] .
$$

The key observation of the Stanley-Reisner theory is that there is a bijective correspondence between squarefree monomial ideals and simplicial complexes.

Definition 2.2 (Stanley-Reisner ideal). The Stanley-Reisner ideal of the simplicial complex $\Delta$ is the monomial ideal

$$
I_{\Delta}=\left\langle x_{\tau}: \tau \notin \Delta\right\rangle .
$$

There is a big advantage of working with symbolic powers of monomial ideals rather than symbolic powers of arbitrary ideals. It follows from the following extremely useful result that one can avoid localizations, see [4, Theorem 3.7] and [1, Theorem 2.5].

Theorem 2.3 (Symbolic powers of monomial ideals). Let $I \subset \mathbb{K}\left[x_{0}, \ldots, x_{N}\right]$ be a monomial ideal with minimal primary decomposition

$$
I=P_{1} \cap \ldots \cap P_{s}
$$

Then, for all $m \geqslant 1$ there is

$$
I^{(m)}=P_{1}^{m} \cap \ldots \cap P_{s}^{m} .
$$

\subsection{Bipyramids}

Following Bocci and Franci [2, for $n \geqslant 3$, we define a bipyramide $B_{n}$ over an $n$-gon $\Gamma_{n}$ as the convex hull of the following set of points

$$
\left\{(0,1),(1,0),(\varepsilon, 0),\left(\varepsilon^{2}, 0\right), \ldots,\left(\varepsilon^{n-1}, 0\right),(0,-1)\right\} \subset \mathbb{C} \times \mathbb{R},
$$


where $\varepsilon$ is a primitive root of 1 of order $n$ and $\Gamma_{n}$ has vertices in the plane $y=0$. Thus a bipyramid is a polytop. Numbering its vertices as follows

$$
P_{0}=(0,1), P_{k}=\left(\varepsilon^{k}, 0\right) \text { for } k=1, \ldots, n, P_{N}=P_{n+1}=(0,-1)
$$

and assigning to each face of $B_{n}$ the set of its vertices, we obtain a simplicial complex with $V=$ $\{0,1, \ldots, N\}$. Thus its Stanley-Reisner ideal is

$$
I_{B_{n}}=\left\langle x_{0} x_{N}, x_{i} x_{j} \text { with } 1 \leqslant i<j \leqslant n \text { and } P_{i} P_{j} \text { not an edge of } \Gamma_{n}\right\rangle .
$$

For $i=1, \ldots, n$, let

$$
S_{i}=\left\{x_{i}, x_{i+1}, \ldots, x_{i+n-2}\right\} \text { and } T_{i}=\left\{x_{i}, x_{i+1}, \ldots, x_{i+n-3}\right\},
$$

where the indices are numbered so that $x_{n+i}=x_{i}$ for $i=1, \ldots, N$. It is easy to check that

$$
I_{B_{n}}=\bigcap_{i=1}^{n}\left\langle x_{0}, T_{i}\right\rangle \cap \bigcap_{i=1}^{n}\left\langle x_{N}, T_{i}\right\rangle
$$

is the primary decomposition of $I_{B_{n}}$, see [2, Proposition 3.2].

Example 2.4. For $n=4$ the bipyramid $B_{4}$ is indicated in Figure 1. We have

$$
\begin{aligned}
& I_{B_{4}}=\left\langle x_{0} x_{5}, x_{1} x_{3}, x_{2} x_{4}\right\rangle \\
& I_{B_{4}}=\left\langle x_{0}, x_{1}, x_{2}\right\rangle \cap\left\langle x_{0}, x_{2}, x_{3}\right\rangle \cap\left\langle x_{0}, x_{3}, x_{4}\right\rangle \cap\left\langle x_{0}, x_{1}, x_{4}\right\rangle \cap \\
& \cap\left\langle x_{1}, x_{2}, x_{5}\right\rangle \cap\left\langle x_{2}, x_{3}, x_{5}\right\rangle \cap\left\langle x_{3}, x_{4}, x_{5}\right\rangle \cap\left\langle x_{1}, x_{4}, x_{5}\right\rangle .
\end{aligned}
$$

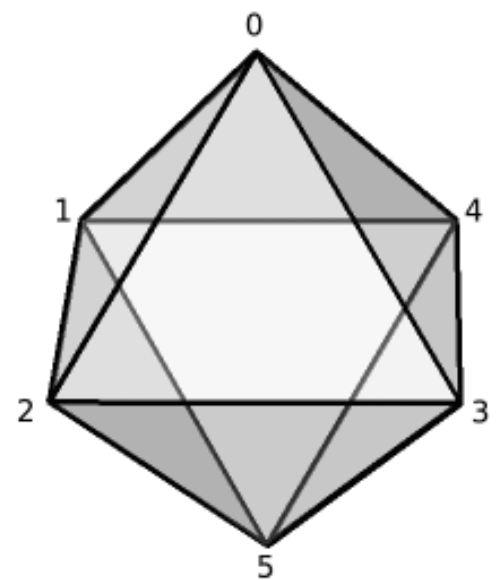

Figure 1: The bipyramid $B_{4}$

The main result of [2] is the following Theorem (2, Theorem 1.1]).

Theorem 2.5. For any $n \geqslant 4$, the Waldschmidt constant of the Stanley-Reisner ideal $I_{B_{n}}$ of a bipyramid $B_{n}$ is

$$
\widehat{\alpha}\left(I_{B_{n}}\right)=\frac{n}{n-2} .
$$

For $n=3$ there is $I_{B_{3}}=\left\langle x_{0} x_{4}\right\rangle$ and hence $\widehat{\alpha}\left(I_{B_{3}}\right)=2$. 
This Theorem has been already reproved in [1, Theorem 6.10], the authors appeal however to fractional chromatic numbers of hypergraphs and use the advanced machinery developed in their paper. We provide here, as an alternative, yet another, fairly elementary proof.

Proof of Theorem 2.5. By definition (4), the Waldschmidt constant is a limit of a sequence, hence it can be computed by an arbitrary subsequence. We use the subsequence indexed by $s(n-2)$ for $s \geqslant 1$.

Our first observation is that

$$
\left(x_{1} \cdot \ldots \cdot x_{n}\right) \in I^{(n-2)} .
$$

Indeed, combining Theorem 2.3 and (6), we see that $I^{(n-2)}$ is the intersection of ideals of the type

$$
\left\langle x_{u}, T_{i}\right\rangle^{(n-2)},
$$

where $u \in\{0, N\}$. Thus, clearly

$$
x_{i} \cdot x_{i+1} \cdot \ldots \cdot x_{i+n-3} \mid x_{1} \cdot \ldots \cdot x_{n} .
$$

Since $\operatorname{deg}\left(x_{1} \cdot \ldots \cdot x_{n}\right)=n$, we have

$$
\widehat{\alpha}\left(I_{B_{n}}\right) \leqslant \frac{n}{n-2} .
$$

Turning to the reverse inequality, assume by the way of contradiction that there is a monomial $f=x_{0}^{a_{0}} \cdot x_{1}^{a_{1}} \cdot \ldots \cdot x_{n+1}^{a_{n+1}}$ of degree $\leqslant s n-1$ in $I^{(s(n-2))}$, i.e.

$$
\sum_{i=0}^{N} a_{i} \leqslant s n-1 .
$$

Since $f$ is contained in all ideals $\left\langle x_{u}, T_{i}\right\rangle^{s(n-2)}$ with $u \in\{0, N=n+1\}$ and $i=1, \ldots, n$, we obtain $2 n$ inequalities of the type

$$
a_{u}+a_{i}+a_{i+1}+\ldots+a_{i+n-3} \geqslant s(n-2) .
$$

Summing these inequalities, we get

$$
n\left(a_{0}+a_{N}\right)+2(n-2) \sum_{i=1}^{n} a_{i} \geqslant 2 n s(n-2) .
$$

Since $n \geqslant 4$, the left hand side is bounded from above by $2(n-2) \sum_{i=0}^{N} a_{i}$. Taking (8) into account, we obtain

$$
2 n s(n-2) \leqslant 2(n-2)(s n-1),
$$

which is a clear contradiction. Hence we conclude that

$$
\frac{\alpha\left(I^{(s(n-2))}\right)}{s(n-2)} \geqslant \frac{s n}{s(n-2)}=\frac{n}{n-2} \text { for all } s \geqslant 1 .
$$

Combining (7) with (10) we obtain the assertion.

\section{Bipyramidal graph}

In this section we consider a graph, rather than a hypergraph, defined by vertices of a bipyramid. To be more precise, we define the bipyramidal graph $D_{n}$ as the set of vertices $V=\left\{P_{0}, P_{1}, \ldots, P_{n}, P_{N}\right\}$ with $N=n+1$ together with the set of edges

$$
E=\left\{P_{0} P_{i}, P_{N} P_{i}, P_{1} P_{2}, P_{2} P_{3}, \ldots, P_{n-1} P_{n}, P_{n} P_{1}, P_{0} P_{N} \text { for } i=1, \ldots, n\right\} .
$$


Example 3.1. For $n=4$ the graph is indicated in Figure 2. We have

$$
\begin{aligned}
& I_{D_{4}}=\left\langle x_{1} x_{3}, x_{2} x_{4}, x_{0} x_{1} x_{2}, x_{0} x_{2} x_{3}, x_{0} x_{3} x_{4}, x_{0} x_{4} x_{1}, x_{1} x_{2} x_{5}, x_{2} x_{3} x_{5}, x_{3} x_{4} x_{5}, x_{4} x_{1} x_{5}\right\rangle \\
& I_{D_{4}}=\left\langle x_{0}, x_{5}, x_{1}, x_{2}\right\rangle \cap\left\langle x_{0}, x_{5}, x_{2}, x_{3}\right\rangle \cap\left\langle x_{0}, x_{5}, x_{3}, x_{4}\right\rangle \cap\left\langle x_{0}, x_{5}, x_{1}, x_{4}\right\rangle \cap \\
& \cap\left\langle x_{1}, x_{2}, x_{3}, x_{5}\right\rangle \cap\left\langle x_{2}, x_{3}, x_{4}, x_{5}\right\rangle \cap\left\langle x_{3}, x_{4}, x_{1}, x_{5}\right\rangle \cap\left\langle x_{1}, x_{2}, x_{4}, x_{5}\right\rangle \cap \\
& \cap\left\langle x_{0}, x_{1}, x_{2}, x_{3}\right\rangle \cap\left\langle x_{0}, x_{2}, x_{3}, x_{4}\right\rangle \cap\left\langle x_{0}, x_{3}, x_{4}, x_{1}\right\rangle \cap\left\langle x_{0}, x_{1}, x_{2}, x_{4}\right\rangle \cap \\
& \cap\left\langle x_{1}, x_{2}, x_{3}, x_{4}\right\rangle .
\end{aligned}
$$

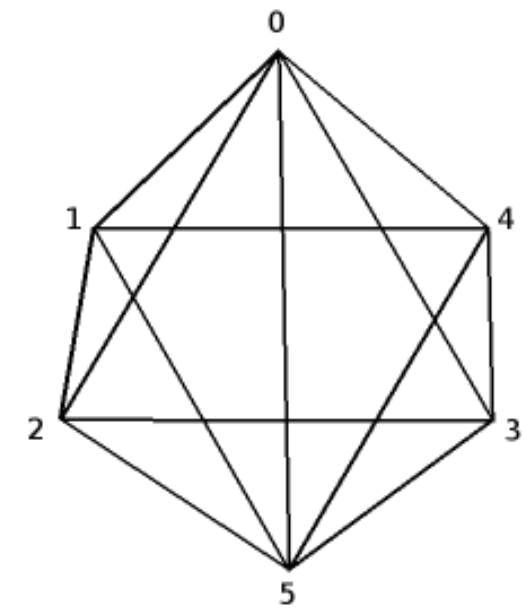

Figure 2: The bipyramidal graph $D_{4}$

Theorem 3.2. For the Stanley-Reisner ideal $I_{D_{n}}$ of the bipyramidal graph $D_{n}$ we have

$$
\widehat{\alpha}\left(I_{D_{n}}\right)=\frac{n+2}{n} .
$$

Proof. Note to begin with that using the notation in (5)

$$
I_{D_{n}}=\bigcap_{i=1}^{n}\left\langle x_{0}, x_{N}, T_{i}\right\rangle \cap \bigcap_{i=1}^{n}\left\langle x_{0}, S_{i}\right\rangle \cap \bigcap_{i=1}^{n}\left\langle x_{N}, S_{i}\right\rangle \cap\left\langle x_{1}, \ldots, x_{n}\right\rangle
$$

is the primary decomposition.

It follows that $x_{0} \cdot x_{N} \cdot x_{1} \cdot \ldots \cdot x_{n} \in I_{D_{n}}^{(n)}$, hence

$$
\widehat{\alpha}\left(I_{D_{n}}\right) \leqslant \frac{n+2}{n} .
$$

Turning to the lower bound, we study the sequence of symbolic powers of $I_{D_{n}}$ indexed by multiples $s n$ of $n$ for $s \geqslant 1$. We assume that there is an $s$ such that $I_{D_{n}}^{(n s)}$ contains a monomial $g=$ $x_{0}^{a_{0}} \cdot x_{N}^{a_{N}} \cdot x_{1}^{a_{1}} \cdot \ldots \cdot x_{n}^{a_{n}}$ of degree

$$
a_{0}+a_{N}+a_{1}+\ldots+a_{n} \leqslant s(n+2)-1 .
$$

Since $g \in\left\langle x_{1}, \ldots, x_{n}\right\rangle$, we also have

$$
a_{1}+\ldots+a_{n} \geqslant s n
$$


It follows from 133 and 14 that

$$
a_{0}+a_{N} \leqslant 2 s-1 .
$$

Since there is in the decomposition (11) an ideal which misses arbitrary two consecutive indices in the set $\{0,1, \ldots, n, N=(n+1)\}$, we obtain by the same token that

$$
a_{i}+a_{i+1} \leqslant 2 s-1 .
$$

for $i=0, \ldots, n$. Summing up altogether $(n+2)$ inequalities in $(15)$ and $(16)$, we obtain

$$
a_{0}+a_{N}+a_{1}+\ldots+a_{n} \leqslant(n+2)\left(s-\frac{1}{2}\right) .
$$

On the other hand, since $g$ is an element in all ideals in the decomposition (11), we obtain, analogously to 14

$$
a_{i}+\ldots+a_{n+i} \geqslant s n .
$$

for all $i=0, \ldots, N$, of course with the convention that the indices are taken modulo $(n+2)$. Summing up these inequalities we get

$$
a_{0}+a_{N}+a_{1}+\ldots+a_{n} \geqslant(n+2) s .
$$

Inequalities (17) and 19 give the desired contradiction, implying that all polynomials in $I_{D_{n}}^{n s}$ have degree al least $s(n+2)$. This, in turn, implies that

$$
\widehat{\alpha}\left(I_{D_{n}}\right)=\lim _{s \rightarrow \infty} \frac{\alpha\left(I_{D_{n}}^{(n s)}\right)}{n s} \geqslant \frac{s(n+2)}{s n}=\frac{n+2}{n} .
$$

Thus 12 and 20 establish the assertion and we are done.

Acknowledgements. Our research was partially supported by National Science Centre, Poland, grant 2014/15/B/ST1/02197.

\section{References}

[1] Bocci, C., Cooper, S., Guardo, E., Harbourne, B., Janssen, M., Nagel, U., Seceleanu, A., Van Tuyl, A., Vu, T.: The Waldschmidt constant for squarefree monomial ideals. J. Algebraic Combin. 44, 875-904 (2016)

[2] Bocci, C., Franci, B.: Waldschmidt constants for StanleyReisner ideals of a class of simplicial complexes. J. Algebra Appl. 15, No. 6, 1650137 (13 pages), (2016)

[3] Bocci, C., Harbourne, B.: Comparing Powers and Symbolic Powers of Ideals. J. Algebraic Geometry 19, 399-417 (2010)

[4] Cooper, S. M., Embree, R. J. D.; Ha, H. T., Hoefel, A. H.: Symbolic powers of monomial ideals. Proc. Edinb. Math. Soc. (2) 60, 3955 (2016)

[5] Ein, L., Lazarsfeld, R., Smith, K.: Uniform bounds and symbolic powers on smooth varieties. Invent. Math. 144, 241-252 (2001)

[6] Francisco, Ch., Ha, H. T., Mermin, J.: Powers of square-free monomial ideals and combinatorics. Commutative algebra, pp. 373-392, Springer, New York (2013)

[7] Francisco, Ch., Mermin, J., Schweig, J.: A survey of Stanley-Reisner theory. Connections between algebra, combinatorics, and geometry, pp. 209-234, Springer Proc. Math. Stat., 76, Springer, New York (2014)

[8] Hochster, M., Huneke, C.: Comparison of symbolic and ordinary powers of ideals. Invent. Math. 147, 349-369 (2002)

[9] Malara, G., Szemberg, T., Szpond, J.: On a conjecture of Demailly and new bounds on Waldschmidt constants in $\mathbb{P}^{N}$. arXiv:1701.04848 
[10] Waldschmidt, M.: Propriétés arithmétiques de fonctions de plusieurs variables II. Séminaire P. Lelong (Analyse), 1975/76, pp. 108-135, Lecture Notes Math. 578, Springer, Heidelberg (1977)

Tomasz Szemberg, Department of Mathematics, Pedagogical University of Cracow, Podchorążych 2, PL-30-084 Kraków, Poland

E-mail address: tomasz.szemberg@gmail.com

Justyna Szpond, Department of Mathematics, Pedagogical University of Cracow, Podchorążych 2, PL-30-084 Kraków, Poland

E-mail address: szpond@up.krakow.pl 